\title{
ESTRUCTURA CRISTALINA DEL COBRE, PROPIEDADES MICROSCÓPICAS MECÁNICAS Y DE PROCESAMIENTO
}

\section{CRYSTALLINE STRUCTURE OF COPPER, MECHANICAL MICROSCOPIC PROPERTIES AND OF PROCESSING}

Fecha de Recepción: 03 de Septiembre de 2006 Fecha de Aprobación: 11 de Octubre de 2006
Jaime Duque Jaramillo ${ }^{1}$ Luis Eduardo Llano Sánchez ${ }^{2}$ Hernando Villazón Amarís ${ }^{3}$

RESUMEN: El objetivo primordial de este artículo es evidenciar la relación entre la estructura cristalina, las propiedades mecánicas a tracción y el procesamiento de una lámina de cobre electrolítico. Luego de una breve descripción teórica sobre las generalidades del cobre y su estructura cristalina, se explican las técnicas de metalografía y de microscopía de transmisión de electrones (TEM), empleadas por los autores para determinar esta estructura. También se evalúan las propiedades mecánicas a tracción y se formulan las relaciones que explican el comportamiento macroscópico del material con relación a su estructura cristalina. Finalmente se sugiere una aplicación del material estudiado en nuevas tecnologías como los son los nano y micro sistemas NEMS y MEMS.

PALABRAS CLAVE: Estructura Cristalina, pruebas metalográficas, granos, dislocaciones, esfuerzo - deformación

ABSTRACT: The main goal of this article is to show the correlation between the crys-talline structure, the mechanical properties to traction and the processing of an electro-lytic copper foil. After a brief theoretical description about copper and its structure, the autors explain the metallographic and electron transmission microscopy (TEM) tech-niques used in the analysis. Additionally, the mechanical properties to traction were evaluated and relationships were formulated to explain the macroscopic behavior of the material related to its crystalline structure. Finally, we suggest an application of this material in new technologies as nano and microsystems NEMS and MEMS.

KEYWORDS: Crystalline structure, metallographic tests, grains, dislocations, strain - stress curve.

1 Ingeniero Mecánico, Universidad Nacional de Colombia. Jefe Laboratorio de Metalografía y Resistencia de Mate-riales, Departamento. Ing. Mecatrónica UMNG. Docente Tiempo completo, Dpto. Ing. Mecatrónica UMNG. E-mail: jaimeduque@umng.edu.co

2 Ingeniero Mecánico, Universidad Nacional de Colombia. Jefe Laboratorio de Tecnología Mecánica UMNG. Do-cente Tiempo completo, Dpto. Ing. Mecatrónica UMNG. Estudiante Maestría en Ing. Mecánica, Universidad de los Andes. E-mail: luis.llano@umng.edu.co

4 Ingeniero Químico, Universidad Nacional de Colombia. Jefe Laboratorio de Térmicas UMNG. Jefe del Area de Materiales, Dpto. Ing. Mecatrónica UMNG. Docente Tiempo completo, Dpto. Ing. Mecatrónica UMNG. E-mail: havillaz@umng.edu.co 


\section{INTRODUCCIÓN}

Con el fin de lograr una mejor comprensión de los comportamientos y las características que ofrece un material, se hace necesario evidenciar la relación entre la estructura cristalina, las propiedades y el procesamiento del mismo. [1]

El estudio de la estructura cristalina del material se realiza teniendo en cuenta la estructura atómica y los tipos de enlaces presentes, la manera como están organizados los átomos, los descriptores utilizados para caracterizar los arreglos estructurales y las simetrías que se hallan presentes. [3] Si bien es posible generar hipótesis a cerca del comportamiento que puede tener el material a nivel macroscópico a partir de las generalidades sobre su estructura, no debemos olvidar que estas surgen de analizar un medio ideal, para el cual no se consideran las imperfecciones presentes en el medio cristalino. [3]

\section{A. Generalidades del cobre}

El cobre es un metal dúctil muy abundante sobre la corteza terrestre, se encuentra mezclado con otros metales $(\mathrm{Au}, \mathrm{Ag}, \mathrm{Bi}$ y $\mathrm{Pb})$ y con sulfuros, sulfatos, carbonatos y óxidos minerales. Tiene poca actividad química: únicamente se oxida lentamente en el aire húmedo recubriéndose de una capa de carbonato básico que es anticorrosivo. Su oxidación se favorece en medios ácidos [5].

Debido a su gran ductilidad y alta conductividad eléctrica, la aplicación industrial más usual es la fabricación de cables para conducción eléctrica, los cuales se pueden fabricar de cualquier diámetro desde $0,025 \mathrm{~mm}$ en adelante. La resistencia de fluencia a la tensión de cables de cobre es aproximadamente $4200 \mathrm{~kg} / \mathrm{cm}^{2}[4]$.

\section{B. Estructura Cristalina}

El cobre puro tiene una red de Bravais del tipo cúbico[1], mostrando una estructura cúbica centrada en las caras FCC (Face Centered Cubic) (Ver Figura 1) en la que se distinguen espacios tetraédricos (Ver Figura 2) .Las figuras se obtienen utilizando el programa JMOL de Java Applet [7]. Los parámetros de red característicos son $a=b=c=3,6151$ Amstrongs y $\beta=90^{\circ}$ [2].

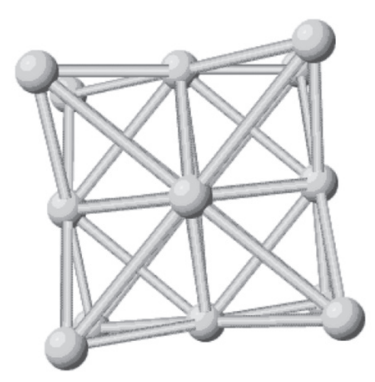

(a)

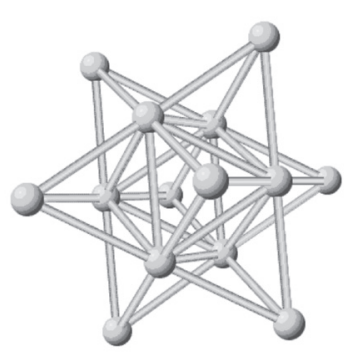

(b)
Figura 1. Estructura Cristalina del Cobre. (a) Celda FCC vista frontal (b) Celda FCC vista lateral

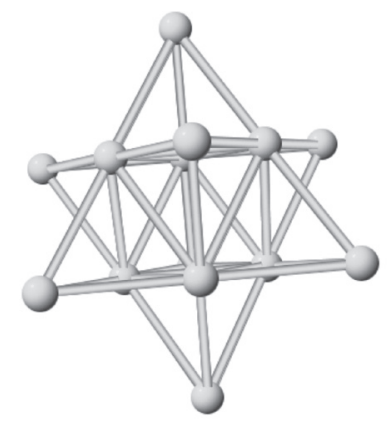

(a)

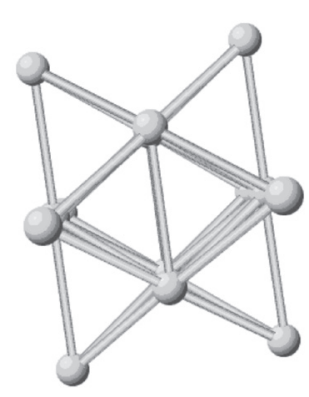

(b)
Figura 2. Espacios tetragonales presentes en la estructura FCC del cobre. a) Vista frontal b) Vista lateral.

La simetría dominante es cúbica axial. [1]. Los planos de deslizamiento preferenciales en este tipo de estructura pertenecen a la familia de planos [111]en las direcciones $<110\rangle$ que corresponde a la dirección más compacta en este tipo de empaquetamiento. [2].

De acuerdo con esta estructura cristalina y las imperfecciones presentes en el medio, se generan comportamientos típicos del material. Es por esta razón que establecer relaciones apropiadas entre estructura, 
propiedades y procesamiento se vuelve una tarea importante al buscar nuevas aplicaciones para el cobre.

\section{MATERIALES Y MÉTODOS}

\section{A. Materiales}

El material estudiado es lámina de cobre electrolítico con una pureza de 99,9\%.

\section{B. Métodos}

El estudio de la estructura cristalina se hace por medio de pruebas metalográficas y de microscopía de transmisión de electrones TEM (Transmision Electron Microscopy).

\section{Metalografía}

La muestra se pule por medio de lijas 200,400 y 600 y después en paño utilizando alúmina como material abrasivo. Posteriormente, la muestra se ataca químicamente con una solución de cloruro férrico al 3\% en volumen. Ensayo realizado en los laboratorios de la Universidad Militar Nueva Granada.

\section{Microscopía de Transmisión de Electrones TEM}

La muestra para análisis tiene un espesor menor a $200 \mu \mathrm{m}$ con el cual se alcanza una región "electrónicamente transparente" para llevar a cabo la observación.

El equipo utilizado es un TECNAI G. en los Laboratorios de la Universidad Nacional de Colombia, en la sede Bogota. Se hace la lectura de la muestra teniendo una inclinación de $\pm 10^{\circ}$ con respecto a la horizontal, temperatura de $18^{\circ} \mathrm{C}$ y un ambiente de vacío de $10^{-4}$ Tor.

\section{Pruebas Mecánicas de Resistencia a la Tracción}

La evaluación de propiedades mecánicas a tensión de la lámina se hizo en una máquina INSTRON a una velocidad de $12,5 \mathrm{~mm} / \mathrm{min}$. En los Laboratorios de la Universidad de los Andes.

\section{RESULTADOS}

El estudio de la microestructura del cobre se llevó a cabo con la ayuda de dos técnicas distintas, la microscopía de transmisión electrónica y por medio de pruebas metalográficas. Por medio de la microscopia de transmisión se evidenciaron las dislocaciones presentes y se vieron algunos granos con diferentes orientaciones, es decir, se observaron estructuras a nivel de una jerarquía del orden de micras.

\section{A. Metalografía}

Por medio de la observación metalográfica se busca ver fases presentes, distribución de fases, granos, tamaño promedio de grano, forma de grano. Sin embargo, al tratarse de un material 99,9\% puro solamente se observa la presencia de una fase continua de cobre. Por esto mismo, no se evidencian fácilmente granos individuales del material al aumento utilizado (100X) (Ver Figura 3).

Por medio de la metalografía solamente se observaron efectos microscópicos de orientación. La Figura 4 fue tomada de la muestra de cobre lijada pero sin ataque químico. En ella se puede observar una orientación preferencial del material en dirección longitudinal debida al proceso de laminación, el cual induce un alargamiento de los granos y orientación en la dirección de laminación. En la imagen también se observan unas líneas oscuras marcadas todas con una misma orientación, resultado del proceso de pulido de la superficie del cobre (material muy blando, dureza Mohs de 2,8. $50-80 \mathrm{HBN}$ ), el cual es muy agresivo y queda con estas impresiones superficiales muy fácilmente [4].

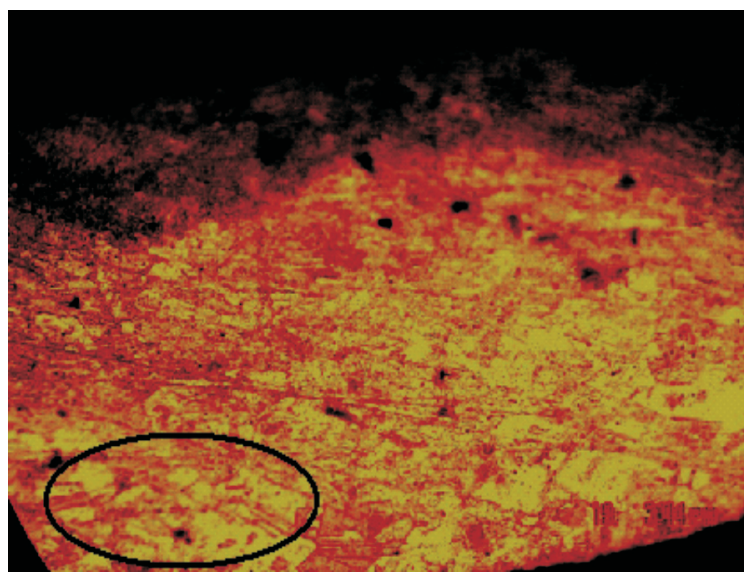

Figura 3. Superficie de cobre. En el círculo se observan granos de material con algún grado de alargamiento y cierta orientación 


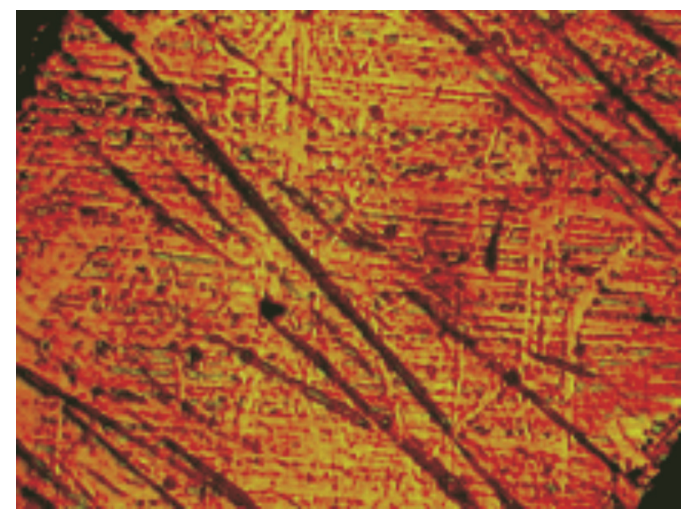

Figura 4. Orientación de granos en lámina de cobre electrolítico. Imagen a $100 \mathrm{X}$

En la Figura 5 se puede apreciar la orientación presente en los sentidos longitudinal y transversal

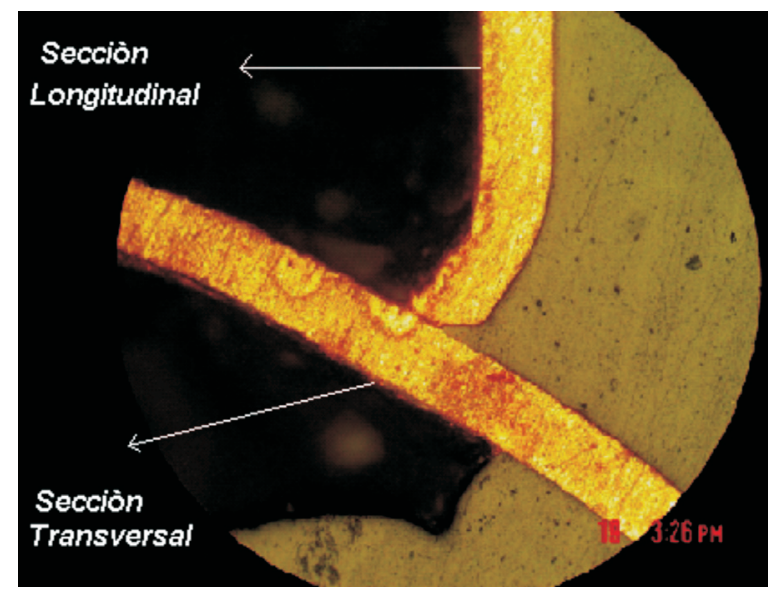

Figura 5. Orientación preferencial de los granos de acuerdo con la dirección de laminado

\section{B. TEM}

Por medio de esta técnica se están evidenciando las imperfecciones presentes en el material. En las imágenes se observan dislocaciones de línea.

A $17000 X$ se identificaron en la figura 6a dos granos de cobre alargados, con cierta orientación. Los defectos podrían haber sido causados por el proceso de laminación, sin embargo no se puede afirmar esto teniendo en cuenta solamente la prueba de TEM.

La figura $6 \mathrm{~b}$ muestra varios granos con diferentes orientaciones, que se evidencian por cambios de color.

La figura 6c muestra un grupo de imperfecciones de línea ubicadas en el contorno de la muestra. Estos defectos se asocian a dislocaciones de borde (conservativas) debidas a la inserción de líneas de átomos en la estructura del cristal.

En la figura $6 \mathrm{~d}$ se observan en el fondo de color verde oscuro las líneas de borde de grano. Las líneas oscuras son dislocaciones formando un posible bosque de dislocaciones.

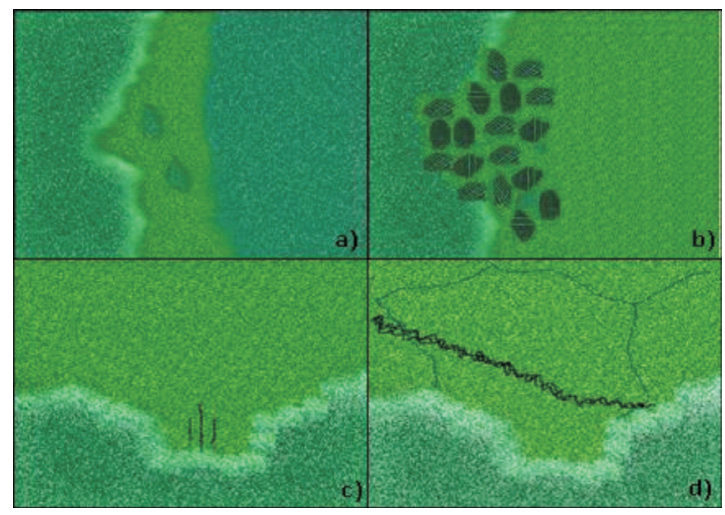

Figura 6 Imágenes obtenidas con el TEM a 17000X para $a$ y $b$ y $55000 X$ para $c$ y d. a) Granos alargados de cobre con algún grado de orientación. b) Orientación de granos de cobre. c) Defectos de línea (3).d) Cordillera de defectos. (Lab. UNC sede bogota)

La Figura 7 ilustra diferentes tipos de dislocaciones encontradas en la muestra. La figura 7a muestra una magnificación de posibles bosques de dislocaciones, que se observan como cordilleras en un entorno continental. Se encuentran también algunas dislocaciones de línea trenzadas (7b), bosques de dislocaciones y unas dislocaciones típicas del cobre recocido las cuales tienen forma de tridente.

Vol. 16 - No. 2 • Diciembre 2006 99 

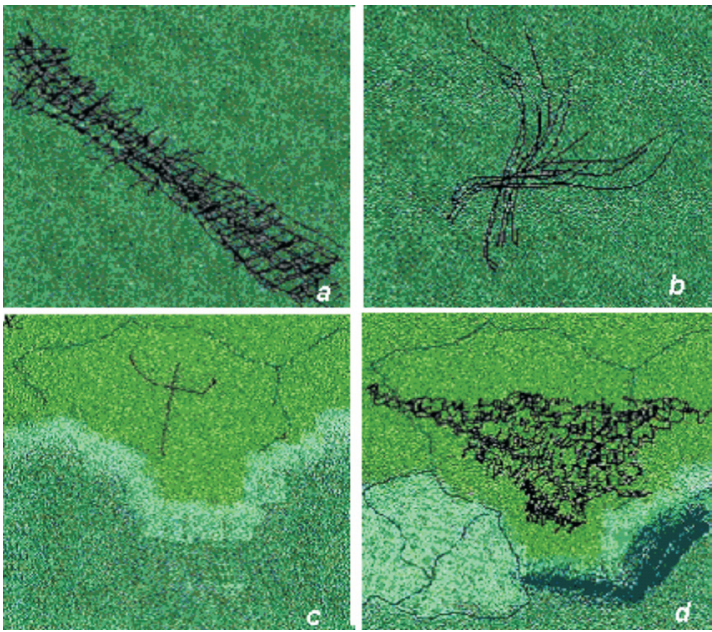

Figura 7. Defectos en el cobre a $55000 X$ para $a, b$ y c y a $25500 X$ para d. a) Magnificación de la cordillera de dislocaciones. b) Ampliación de dislocaciones de línea trenzadas. c) Defecto típico de cobre recocido (tridente). d) Bosque de dislocaciones. Bordes de grano. Franjas de curvatura. (Lab. UNC sede Bogotá)

La figura 7c se observa un tridente conformado por dos dislocaciones de borde conectadas por una dislocación de borde inicial. Este tipo de defecto es típico del $\mathrm{Cu}$ recocido y probablemente también se debe presentar en materiales con estructura cristalina FCC.

La Figura 7d es muy interesante dado que en esta imagen se pueden observar simultáneamente un gran bosque de dislocaciones junto con bordes de grano y "estrías". Las estrías son franjas de contorno en forma de palomas que se producen por acumulaciones de átomos que no han tenido el tiempo suficiente de difundirse y reorganizarse para recuperar la estructura cristalina. Estas probablemente se produjeron por la diferencia de espesores en la muestra y el tratamiento térmico.

\section{Pruebas de Tensión}

Estos ensayos fueron realizados en los Laboratorios de la Universidad de los Andes.
Los datos obtenidos para las probetas longitudinales se presentan en la Tabla 1, mientras que los correspondientes a probetas transversales, se muestran en la Tabla 2.

Los valores de carga y elongación máxima y módulo de elasticidad, están incluidos en las tablas.

Tabla 1. Datos de tracción para las probetas en dirección longitudinal

\begin{tabular}{|c|c|c|c|c|c|c|}
\hline \multicolumn{7}{|c|}{ LONGITUDINAL } \\
\hline Probeta & & 1 & 2 & 3 & 4 & 5 \\
\hline $\begin{array}{l}\text { Finalización } \\
\text { Ancho } \\
\text { Espesor }\end{array}$ & $\begin{array}{l}\mathrm{mm} \\
\mathrm{mm}\end{array}$ & \begin{tabular}{|c|} 
Ruptura \\
13.25000 \\
0.19050
\end{tabular} & \begin{tabular}{|c|} 
Ruptura \\
13.20000 \\
0.19050 \\
\end{tabular} & $\begin{array}{c}\text { Ruptura } \\
13.40000 \\
0.19050\end{array}$ & \begin{tabular}{|c|} 
Ruptura \\
13.40000 \\
0.19050
\end{tabular} & $\begin{array}{r}\text { Ruptura } \\
13.30000 \\
0.19050\end{array}$ \\
\hline $\begin{array}{l}\text { Area } \\
\text { Longitud Ext. Gauge } \\
\text { Longitud Spec gauge }\end{array}$ & $\begin{array}{l}\mathrm{mm} 2 \\
\mathrm{~mm} \\
\mathrm{~mm}\end{array}$ & \begin{tabular}{r|}
2.52412 \\
50.00000 \\
57.00000
\end{tabular} & \begin{tabular}{|l|}
2.51460 \\
50.00000 \\
57.00000
\end{tabular} & $\begin{array}{c}2.55270 \\
50.00000 \\
57.00000\end{array}$ & $\begin{array}{r}2.55270 \\
50.00000 \\
57.00000\end{array}$ & $\begin{array}{r}2.53365 \\
50.00000 \\
57.00000\end{array}$ \\
\hline $\begin{array}{l}\text { Puntos } \\
\text { Carga máxima } \\
\text { Extension máxima } \\
\text { Punto de carga máxima }\end{array}$ & $\begin{array}{l}\mathrm{kN} \\
\mathrm{mm}\end{array}$ & \begin{tabular}{|c|}
203 \\
0.47261 \\
6.13076 \\
171 \\
\end{tabular} & \begin{tabular}{|c|}
205 \\
0.47758 \\
6.79738 \\
157 \\
\end{tabular} & $\begin{array}{c}146 \\
0.44563 \\
4.22907 \\
96\end{array}$ & \begin{tabular}{|c|}
138 \\
1.44463 \\
3.83294 \\
88 \\
\end{tabular} & $\begin{array}{c}116 \\
0.43927 \\
2.83509 \\
83 \\
\end{array}$ \\
\hline $\begin{array}{l}\text { Esfuerzo máximo } \\
\text { Modulo } \\
\text { Modulo Promedio }\end{array}$ & $\begin{array}{l}\text { Mpa } \\
\text { Mpa } \\
\text { Mpa }\end{array}$ & \begin{tabular}{|l|}
187.2372 \\
50739.61
\end{tabular} & \begin{tabular}{|l|}
189.9229 \\
42773.09
\end{tabular} & \begin{tabular}{|l|}
174.5720 \\
37119.02 \\
47188.57
\end{tabular} & \begin{tabular}{|l|}
565.9224 \\
22289.67
\end{tabular} & \begin{tabular}{|l}
173.3744 \\
83021.45
\end{tabular} \\
\hline
\end{tabular}

Tabla 2. Datos de tracción para las probetas en dirección transversal

\begin{tabular}{|c|c|c|c|c|c|c|}
\hline \multicolumn{7}{|c|}{ TRANSVERSAL } \\
\hline Probeta & & 6 & 7 & 8 & 9 & 10 \\
\hline \begin{tabular}{|l|} 
Finalización \\
Ancho \\
Espesor \\
\end{tabular} & $\begin{array}{l}\mathrm{mm} \\
\mathrm{mm}\end{array}$ & \begin{tabular}{|c|} 
Ruptura \\
13.25000 \\
0.19050 \\
\end{tabular} & \begin{tabular}{|c|} 
Ruptura \\
13.30000 \\
0.19050 \\
\end{tabular} & \begin{tabular}{|r|} 
Ruptura \\
13.30000 \\
0.19050 \\
\end{tabular} & \begin{tabular}{|c|} 
Ruptura \\
13.30000 \\
0.19050 \\
\end{tabular} & $\begin{array}{c}\text { Ruptura } \\
13.30000 \\
0.19050 \\
\end{array}$ \\
\hline \begin{tabular}{|l|} 
Area \\
Longitud Ext. Gauge \\
Longitud Spec gauge \\
\end{tabular} & $\begin{array}{l}\mathrm{mm} 2 \\
\mathrm{~mm} \\
\mathrm{~mm}\end{array}$ & \begin{tabular}{|c|}
2.52412 \\
50.00000 \\
57.00000 \\
\end{tabular} & \begin{tabular}{|c|}
2.53365 \\
50.00000 \\
57.00000 \\
\end{tabular} & \begin{tabular}{|c|}
2.53365 \\
50.00000 \\
57.00000 \\
\end{tabular} & \begin{tabular}{|c|}
2.53365 \\
50.00000 \\
57.00000 \\
\end{tabular} & $\begin{array}{c}2.53365 \\
50.00000 \\
57.00000 \\
\end{array}$ \\
\hline \begin{tabular}{|l} 
Puntos \\
Carga máxima \\
Extension máxima \\
Punto de carga máxima \\
\end{tabular} & $\begin{array}{l}\mathrm{kN} \\
\mathrm{mm}\end{array}$ & \begin{tabular}{c|}
168 \\
0.44315 \\
5.25243 \\
127 \\
\end{tabular} & \begin{tabular}{c|}
159 \\
0.40063 \\
4.67269 \\
97 \\
\end{tabular} & \begin{tabular}{|c|}
155 \\
0.41939 \\
4.25807 \\
102 \\
\end{tabular} & $\begin{array}{c}241 \\
0.41300 \\
7.51514 \\
97 \\
\end{array}$ & $\begin{array}{c}166 \\
0.42155 \\
4.78901 \\
117 \\
\end{array}$ \\
\hline $\begin{array}{l}\text { Esfuerzo máximo } \\
\text { Modulo }\end{array}$ & $\begin{array}{l}\text { Mpa } \\
\text { Mpa }\end{array}$ & \begin{tabular}{|l|}
175.5658 \\
35563.33 \\
\end{tabular} & \begin{tabular}{|l|}
158.1237 \\
46800.25 \\
\end{tabular} & \begin{tabular}{|l|}
165.5280 \\
34998.13 \\
\end{tabular} & $\begin{array}{r}163.0059 \\
100257.13 \\
\end{array}$ & $\begin{array}{l}166.3805 \\
89729.76 \\
\end{array}$ \\
\hline Modulo Promedio & Mpa & & & 61469.72 & & \\
\hline
\end{tabular}

La Tabla 3 muestra los valores de resiliencia, tenacidad y porcentajes de elongación y de reducción de área obtenidos

Tabla 3. Valores de resiliencia y tenacidad

\begin{tabular}{|c|c|c|c|c|c|c|c|c|c|c|}
\hline PROBETA & L1 & L2 & $\mathrm{L} 3$ & $\mathrm{~L} 4$ & L5 & T1 & $\mathrm{T} 2$ & T3 & $\mathrm{T} 4$ & $\mathrm{T5}$ \\
\hline Resiliencia $\left(\mathrm{MJ} / \mathrm{m}^{2}\right)$ & 0.23 & 0.31 & 0.23 & 5.27 & 0.24 & 0.30 & 0.37 & 0.15 & 0.36 & 0.34 \\
\hline Tenacidad(M $\left(\mathrm{J}^{\prime} \mathrm{m}^{3}\right)$ & 17.48 & 17.00 & 7.65 & 5.18 & 5.14 & 11.77 & 6.78 & 7.15 & 6.66 & 9.00 \\
\hline Elongación (\%) & 9.65 & 9.94 & \begin{tabular}{l|l}
10.04 & 8 \\
\end{tabular} & 8.86 & 6.50 & 9.94 & 10.33 & 11.12 & 7.97 & 6.40 \\
\hline Reducción de área (\%) & 14.77 & 10.20 & \begin{tabular}{l|l|l}
9.10 & 9 \\
\end{tabular} & 9.10 & 8.42 & 8.08 & 10.88 & 8.42 & 8.42 & 8.77 \\
\hline & \multicolumn{3}{|c|}{ Promedio } & & $\mathrm{L}$ & $\mathrm{T}$ & & & & \\
\hline & \multicolumn{3}{|c|}{ Resiliencia $\left(\mathrm{MJ}^{\prime} \mathrm{m}^{2}\right)$} & & 26 & 0.31 & & & & \\
\hline & \multirow{2}{*}{\multicolumn{3}{|c|}{\begin{tabular}{|l|} 
Tenacidad $\left(\mathrm{MJ} / \mathrm{m}^{3}\right)$ \\
Elongación
\end{tabular}}} & & 0.69 & 8.27 & & & & \\
\hline & & & & & 1.00 & 9.15 & & & & \\
\hline & \multicolumn{3}{|c|}{\begin{tabular}{|l|} 
Elongación (\%) \\
Reducción de área (\%) \\
\end{tabular}} & & 0.32 & 8.91 & & & & \\
\hline
\end{tabular}


Las gráficas obtenidas para los ensayos de tensión en las probetas longitudinales y transversales se presentan a continuación en las Figuras 8 y 9 respectivamente.

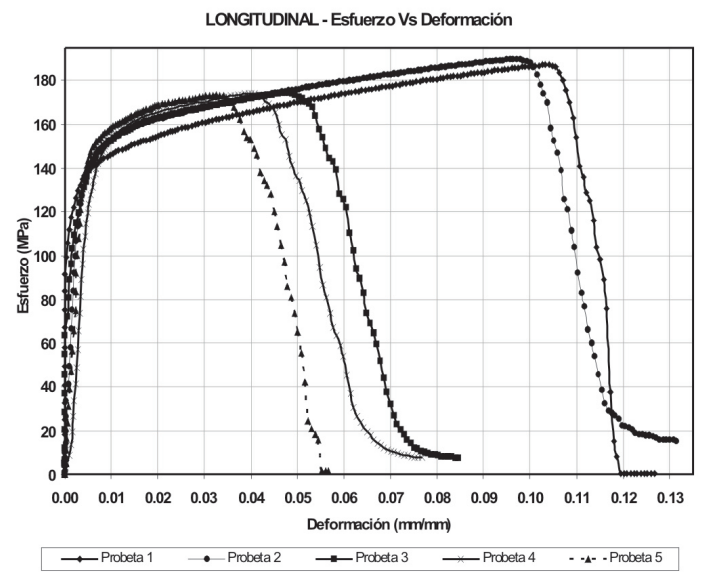

Figura 8. Gráfica Esfuerzo-Deformación Probetas longitudinales

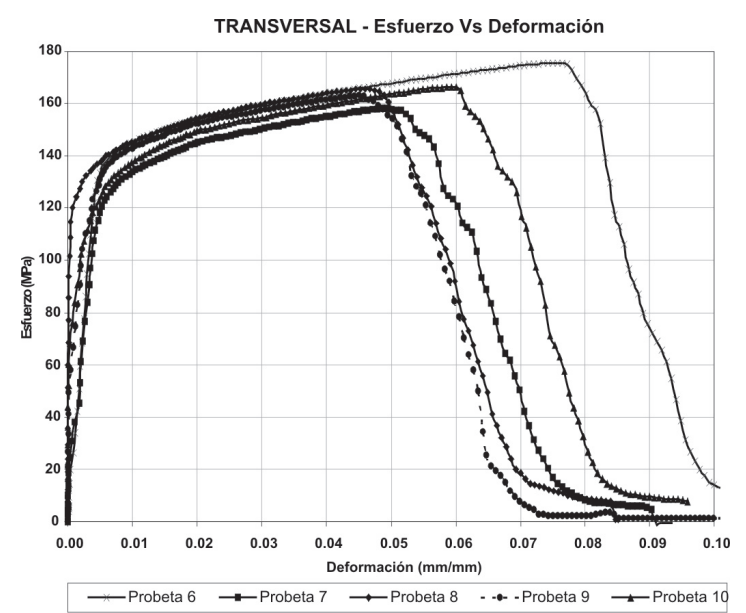

Figura 9. Gráfica Esfuerzo - Deformación probetas transversales

\section{ANÁLISIS Y DISCUSIÓN}

\section{A. Estructura vs. Propiedades}

Con base en el comportamiento exhibido por el material al ser sometido a tracción, se hacen evidentes las diferentes jerarquías estructurales del material.
En la Figura 10 se evidencia la elevada capacidad de almacenamiento de energía del material, presentándose en la macroestructura altas deformaciones plásticas, aunque el material no presente una alta resiliencia. Este macro efecto surge como consecuencia de la estructura interna del material y de la activación de sistemas de deslizamiento.

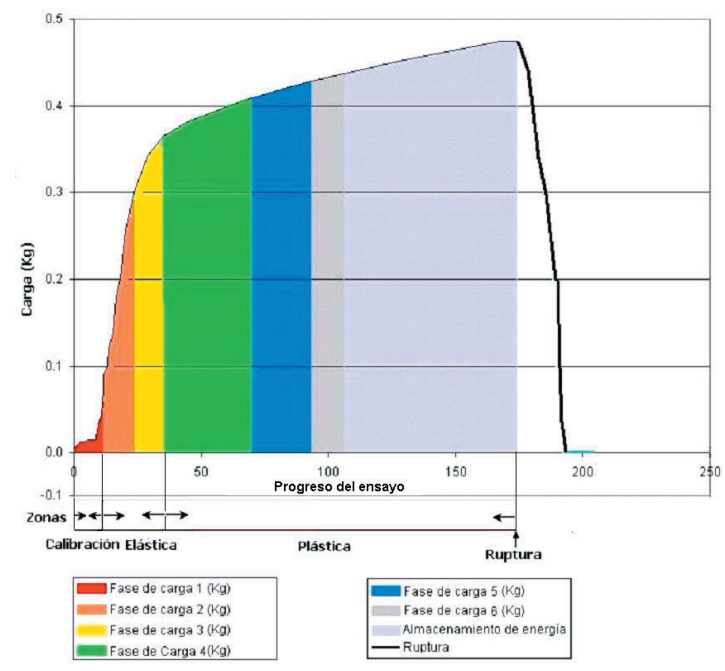

Figura 10. Gráfico comparativo de carga aplicada vs. avance del en-sayo para la probeta 1 en dirección longitudinal

Durante el proceso de aplicación de carga a tensión sobre el material se observan etapas sucesivas a través de las cuales se pueden distinguir varios efectos.

Al principio en la etapa de calibración, los extensómetros no registran valores positivos, no se presenta ninguna deformación, en este momento el material está almacenando energía como potencial en los enlaces atómicos (metálicos covalentes y secundarios) y en los planos más densamente empacados, preparándose para empezar una etapa de deformación elástica.

La zona de deformación elástica es estrecha ya que al ser un material 99,9\% puro no tiene una cantidad importante de imperfecciones que aumente la can- 
tidad de energía requerida para empezar a causar movimientos atómicos. En estas dos primeras prevalece la jerarquía nanocroscópica del material.

La movilización de dislocaciones en la estructura está relacionada con la activación de sistemas de deslizamiento, de la familia (111) característica de la estructura FCC del cobre, la cual se hace presente en la etapa de deformación plástica. Allí se inicia el movimiento de las dislocaciones de línea y de tornillo, causando un efecto macroscópico de deformación. La zona de deformación plástica es amplia debido a que la movilización de dislocaciones se facilita por causa de la pureza del material, la cual evita la presencia de fenómenos de endurecimiento por solución y por precipitación.

La fractura es un efecto macroscópico que es el resultado de la finalización de los procesos de deformación plástica debido a que las dislocaciones ya no se pueden mover más.

En la Figura 11 se pueden observar las zonas mencionadas.

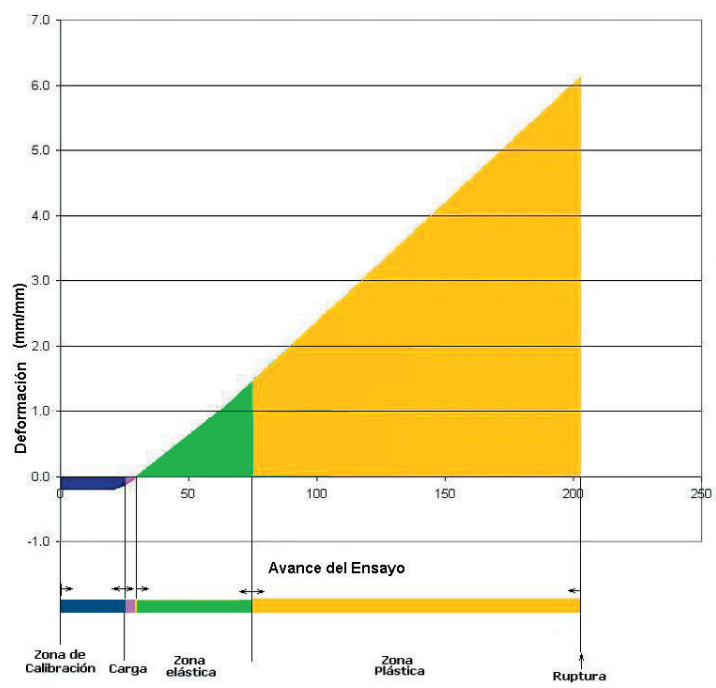

Figura 11. Gráfico comparativo de deformación vs. avance del ensayo para la probeta 1 en dirección longitudinal.

\section{B. Propiedades vs. Procesamiento}

El cobre debido a su estructura atómica y organización espacial posee una alta capacidad de deformación plástica, por lo cual para su procesamiento se utilizan preferiblemente métodos de deformación plástica como el trefilado y el laminado. Estas propiedades, además de su conductividad y abundancia en la naturaleza, permiten que sea muy utilizado en cables de conducción eléctrica.

\section{Estructura-Propiedades -Procesamiento 1. Aplicaciones}

En las nuevas tendencias de miniaturización de componentes electrónicos y sensórica el cobre es utilizado normalmente como electrodo en procesos de electroerosión y electro-deposición [6], sin embargo no es utilizado como componente.

En tecnologías de micro y nano sistemas MEMS y NEMS respectivamente, el cobre podría ser estudiado para ser utilizado como soporte, especialmente en NEMS donde se requiere una mayor versatilidad de los materiales debido a la alta especificidad de la aplicación. Las características del cobre electrolítico como la casi idealidad de estructura cristalina, la conductividad eléctrica que posee y la facilidad de su manufactura, son muy deseables para los soportes [3]; sin embargo, presenta problemas con respecto a la estabilidad mecánica, por su alta ductilidad, con el coeficiente de expansión térmica, y la histéresis mecánica. Si se dopara el material con elementos que contribuyan a mejorar estas falencias, el cobre podría emplearse como sustrato en NEMS. La estructura cristalina del cobre es bastante apta para el dopaje, ya que tiene sitios tetraédricos donde se pueden alojar diversos tipos de átomos.

En la Figura 12 se presenta un espectrograma cualitativo del cobre electrolítico 99,9\% puro, allí se ve que aunque tiene un alto grado de pureza puede contener diversas impurezas en su estructura, las cuales pueden ser seleccionadas a propósito de acuerdo con la necesidad. 
Otra alternativa para mejorar las propiedades del cobre como sustrato podría ser por medio de aleaciones con zinc produciendo latones, y con estaño para obtener bronces, de esta manera se obtiene un material mecánicamente más estable al aumentar su dureza [4]. El oro es muy utilizado como material en NEMS, este tiene propiedades térmicas, eléctricas y de dureza muy similares a las del cobre, sin embargo la principal diferencia radica en los mecanismos de oxidación de los materiales. Mejorando esta característica, el cobre puede llegar a ser una buena alternativa en este tipo de aplicación.

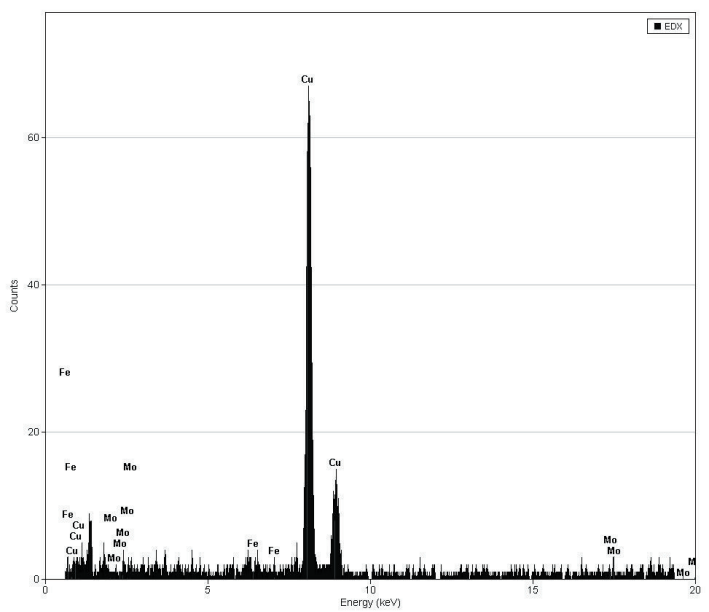

Figura 12. Difractograma cualitativo de composición del cobre electrolítico con 99,9\% de pureza. (Lab. UNC sede bogota)

\section{CONCLUSIONES}

El cobre es un material muy apropiado para aplicaciones donde se vean involucrados procesos de deformación plástica y se requiera un gran volumen de producción porque su obtención y manufactura son de bajo costo.

Para aplicaciones en NEMS el cobre puede ser una alternativa viable, ya que es un material que ha sido estudiado durante mucho tiempo y sus modificaciones y mejoras pueden resultar más simples que en otros materiales.

Debido a la anisotropía y a las propiedades térmicas y eléctricas que presenta el cobre puede ser utilizado en NEMS para sensar propiedades en diferentes direcciones.

\section{REFERENCIAS}

[1] ALLEN S, THOMAS E. "The Structure of materials". MIT series in materials science and engineering; Wiley, New York, 1998.

[2] ASKELAND D. "Ciencia e Ingeniería de los materiales". Editorial Thomson. 1998.

[3] HSU TAI- RAN. MEMS and Microsystems: Design and Manufacture

[4] LAJTIN YU. M.: Metalografía y Tratamiento Térmico de los metales. Editorial MIR. Segunda Edición. Moscú.

[5] Recopilado el 23 de mayo de 2005 de: http://herramientas.educa.madrid.org/tabla/7isotopos/cu7. html

[6] Recopilado el 23 de mayo de 2005 de: http://gauss.ffii.org/PatentView/EP972284

[7] Recopilado el 23 de mayo de 2005 de: http://cst-ww.nrl.navy.mil/lattice/struk/a1.html 